\title{
On a convection-diffusion equation with partial diffusivity *
}

\author{
ANDREA PASCUCCI \\ Dipartimento di Matematica, Università di Bologna ${ }^{\dagger \ddagger}$
}

\begin{abstract}
We consider the Cauchy problem for the nonlinear degenerate equation in $\mathbb{R}^{N+1}$

$$
\operatorname{div}(A \nabla u)+u(\mathbf{b} \cdot \nabla u)-\partial_{t} u=f(\cdot, u)
$$

where $A \geq 0$ is a constant symmetric matrix and $\operatorname{ker}(A)$ is generated by $\mathbf{b}$. We prove the existence of a local viscosity solution $u$ and we study the interior regularity of $u$ in the framework of Hörmander type operators.
\end{abstract}

\section{Introduction}

We consider the Cauchy problem for the nonlinear convection-diffusion equation

$$
\left.\operatorname{div}(A \nabla u)+u(\mathbf{b} \cdot \nabla u)-\partial_{t} u=f(\cdot, u), \quad \text { in } S_{T} \equiv \mathbb{R}^{N} \times\right] 0, T[,
$$

with initial datum

$$
u(\cdot, 0)=g, \quad \text { in } \mathbb{R}^{N} .
$$

We assume that $f, g$ are globally Lipschitz continuous functions. Moreover we assume that the matrix $A$ is constant, symmetric and positive semidefinite. The convection direction $\mathbf{b}$ is constant and generates $\operatorname{ker}(A)$.

Equations of form (1.1) were studied by Escobedo, Vazquez and Zuazua [11] in order to describe the asymptotic behaviour as $t \rightarrow \infty$ of solutions to a related parabolic equation with complete diffusion. We remark that, without loss of generality, by performing a suitable change of variables we may assume that $A$ is diagonal so that $\mathbf{b}$ points along a coordinate axis, for instance $\mathbf{b}=e_{N}$. Then it is convenient to denote a point in $\mathbb{R}^{N}$ by $(x, y)$ with $x=\left(x_{1}, \ldots, x_{N-1}\right)$ and $y \in \mathbb{R}$. Hence (1.1) becomes

$$
L u \equiv \triangle_{x} u+u \partial_{y} u-\partial_{t} u=f(\cdot, u), \quad \text { in } S_{T},
$$

where $\triangle_{x}$ denotes the Laplace operator acting in the $x$ variables.

Equation (1.3) also arises in mathematical finance, when studying agents' decisions under risk. The classical approach for this financial problem is based on the representation of agents'

\footnotetext{
*Keywords: nonlinear degenerate parabolic equation, interior regularity, Hörmander operators.

${ }^{\dagger}$ Piazza di Porta S. Donato 5, 40127 Bologna (Italy). E-mail: pascucci@dm.unibo.it

${ }^{\ddagger}$ Investigation supported by the University of Bologna. Funds for selected research topics.
} 
preferences in the framework of the utility theory and various models have been proposed, aiming to taking into account many aspects of the dynamics of the economy. Epstein and Zin in [10] propose a utility functional which is the solution of a backward stochastic differential equation. Recently Antonelli, Barucci and Mancino [1] propose a more sophisticated utility functional that considers some other aspects of decision making, such as the agents' habit formation, which is described as a smoothed average of past consumption and expected utility. In that model the couple of processes utility and habit is described by a system of backward-forward stochastic differential equations. In [1] is proved that there exists a unique solution $u$ of such system, that satisfies some suitable initial and final conditions, and which is a viscosity solution, in the sense of the User's guide [9] of Cauchy problem (1.3)-(1.2). Moreover, in [1] it is proved that the solution $u$ is defined in a suitably small interval of time $[0, T[$ and satisfies

$$
\begin{aligned}
\left|u(x, y, t)-u\left(x^{\prime}, y^{\prime}, t\right)\right| & \leq c_{0}\left(\left|x-x^{\prime}\right|+\left|y-y^{\prime}\right|\right), \\
\left|u(x, y, t)-u\left(x, y, t^{\prime}\right)\right| & \leq c_{0}(1+|(x, y)|)\left|t-t^{\prime}\right|^{\frac{1}{2}},
\end{aligned}
$$

for every $(x, y),\left(x^{\prime}, y^{\prime}\right) \in \mathbb{R}^{N}, t, t^{\prime} \in[0, T]$, where $c_{0}$ is a positive constant that depends on the Lipschitz constants of $f$ and $g$.

Related problems also arise in stochastic control theory. For instance, the value function $v$ of a suitable control problem is a semiconcave solution of the following Cauchy problem

$$
\begin{aligned}
\partial_{x x} v+\frac{1}{2}\left(\partial_{y} u\right)^{2}-\partial_{t} v & =\varphi, & & \text { in } \left.\mathbb{R}^{2} \times\right] 0, T[, \\
v(\cdot, 0) & =\psi, & & \text { in } \mathbb{R}^{2},
\end{aligned}
$$

for some continuous functions $\varphi$ and $\psi$ (cf. [12]). Note that the function $u=\partial_{y} v$ is, at least formally, a solution of a Cauchy problem like (1.3)-(1.2), even if it is only a locally bounded function.

In this paper we are interested in the existence and interior regularity of local solutions to the Cauchy problem (1.3)-(1.2). Our main results are stated in the next section.

Acknowledgments. The existence Theorem 2.1 is proved independently in a joint work with Antonelli [2] and in [22] in collaboration with Polidoro. The interior regularity problem is studied in [7], [8] with Citti and Polidoro in dimension three, and in [21] in the general case.

\section{Main results}

Our aim is to find a functional space where this problem is well posed. The main difficulty is the mixed parabolic-hyperbolic feature of equation (1.3) due to the lack of diffusion in the $y$ direction, so that it may include the Burgers' equation, when $f \equiv 0$ and $g=g(y)$. We explicitly note that the nonlinearity in (1.3) is not monotone, then a standard comparison principle does not hold and, as a consequence, the uniqueness of the solution is not guaranteed. This fact also affects the existence of the solution. Indeed, when using the classical Bernstein's method, a maximum principle for the operator $L v+v^{2}$ (that occurs when we differentiate both sides of (1.3) w.r.t. $y$ ) is required. Yet also more sophisticated versions of that method (cf. Barles [3]) do not seem to work in our setting. On the other hand, in the space of functions characterized 
by conditions (1.4) the operator $L$ in (1.3) does satisfy a comparison principle. Then we are able to prove the existence of strong solution of the Cauchy problemfor small times. More precisely, we have

Theorem 2.1 Let $f, g$ globally Lipschitz continuous. If $T>0$ is suitably small, then there exists a unique function $u$, verifying estimates (1.4) on $\bar{S}_{T}=\mathbb{R}^{N} \times[0, T]$ and assuming the initial datum $g$, such that

$$
u \in H_{l o c}^{1}\left(S_{T}\right), \quad \triangle_{x} u \in L_{l o c}^{2}\left(S_{T}\right),
$$

and equation (1.3) is satisfied a.e.

Let us remark that, in general, the linear growth of the initial datum $g$ does not allow solutions which are defined globally in $t$. Indeed, let us consider the following simple example: for $N=2$, take $f \equiv 0$ and $g(x, y)=x+y$. A direct computation shows that $u(x, y, t)=\frac{x+y}{1-t}$ is the unique solution to (1.3)-(1.2) and it blows up as $t \rightarrow 1$.

Our main results regard the interior regularity of the strong solution $u$ of Theorem 2.1. Since $L$ is a degenerate second order operator, the known results by Cabre e Caffarelli [5], Trudinger [24], Bian e Dong [4], Wang [25] do not apply. Therefore we set the problem in the framework of subelliptic operators on nilpotent Lie groups. We remark that $L$ is an operator such that the matrix of the coefficients of the second order derivatives is only positive semi-definite. As one can expect, the solution $u$ of the equation $L u=0$ is smooth in the directions in which the matrix is non-degenerate, but not in other directions. Consider for example the operator

$$
L_{1} \equiv \partial_{x}^{2}+\partial_{y}^{2}
$$

in the variables $(x, y, t) \in \mathbb{R}^{3}$. Every solution $u$ of $L_{1} u=0$ is smooth with respect to the variables $x$ and $y$, but is not regular in the variable $t$. However, as Hörmander pointed out in the celebrated paper [16], there are other "regularity directions" for the solution $u$, and these directions are the ones of the commutators. For instance, let us consider the Kohn-Laplace operator in $\mathbb{R}^{3}$ :

$$
L_{2} \equiv\left(\partial_{x}+2 y \partial_{t}\right)^{2}+\left(\partial_{y}-2 x \partial_{t}\right)^{2} .
$$

As before, there are only two directional derivatives, while the dimension of the space is three, but in this case every solution of $L_{2} u=0$ is smooth, not only in the directions of the derivatives $X=\partial_{x}+2 y \partial_{t}$ and $Y=\partial_{y}-2 x \partial_{t}$, but also in the direction of their commutator

$$
[X, Y]=X Y-Y X=-4 \partial_{t} .
$$

The operator considered above is a simple but meaningful example of the class studied by Hörmander in [16]. Let $X_{0}, \ldots, X_{p}$ be a set of linear first order operators (i.e. vector fields) defined as

$$
X_{j}=\sum_{i=1}^{n} a_{i j}(x) \partial_{x_{i}} \quad j=0, \ldots, p
$$

where $a_{i j}$ are smooth functions on some domain $\Omega \subset \mathbb{R}^{n}$ and let also $f \in C^{\infty}(\Omega)$. Hörmander proved in [16] that if $u$ is a solution of the equation

$$
\sum_{i, j=1}^{p} X_{i} X_{j} u+X_{0} u=f, \quad \text { in } \Omega,
$$


and the Lie algebra generated by the vector fields $X_{0}, \ldots, X_{p}$ has rank $n$ at every point of $\Omega$, then $u \in C^{\infty}(\Omega)$.

Hörmander's result was the starting point of an extensive research aiming to investigate the regularity properties of the operators in (2.3) and their links with some suitable Lie group structures on $\mathbb{R}^{n}$. The existence of a fundamental solution and of a control distance have been established in [20], [23], [17]. Using these properties, a general theory of the regularity both in Sobolev spaces and in spaces of Hölder continuous functions has been settled down in [13], [14], [23] and [18].

Aiming to use the linear theory for the study of our problem we can try to consider the "linearized" operator

$$
L_{u}=\triangle_{x}+u \partial_{y}-\partial_{t},
$$

where $u$ is considered as a coefficient, but we immediately realize that the smoothness of the coefficients $a_{i j}$ is a fundamental assumption in the previous papers and, in our problem, we cannot assume that the coefficient $u$ of the equation is $C^{\infty}$, since the smoothness of the solution $u$ is exactly the goal of our study.

Actually, in all the papers cited above it is crucial that the vector fields $X_{j}$ are regular at least as it is sufficient to obtain, by commutation, $n$ linearly independent vector fields at every point of $\mathbb{R}^{n}$. For the first time, Franchi and Lanconelli [15] studied the properties of a control distance related to a family of non regular vector fields, aiming to adapt the classical Moser's iteration scheme to prove the Hölder regularity of weak solutions of an equation of the form

$$
\sum_{i, j=1}^{n} \alpha_{i j}(x) \partial_{x_{i} x_{j}}
$$

where the matrix $\left(\alpha_{i j}(x)\right)$ is positive semi-definite for every $x \in \mathbb{R}^{n}$. Franchi e Lanconelli assume that the operator is "elliptic" w.r.t. a family $X_{1}, \ldots, X_{p}$ of Lipschitz continuous vector fields, in the sense that

$$
\frac{1}{\lambda} \sum_{j=1}^{p}\left\langle X_{j}(x), \xi\right\rangle^{2} \leq\langle A(x) \xi, \xi\rangle \leq \lambda \sum_{j=1}^{p}\left\langle X_{j}(x), \xi\right\rangle^{2},
$$

for some positive constant $\lambda$. This class of operators includes, for instance, in $\mathbb{R}^{2}$

$$
\partial_{x}^{2}+|x|^{2 \alpha} \partial_{y}^{2}
$$

where $\alpha$ is a positive constant. We stress that a general theory for operators with non-smooth coefficients is not available.

Here we employ a technique introduced by Citti in [6] where the author considers the regularity of the solutions to the following equation of prescribed Levi curvature

$$
\mathcal{L} u \equiv u_{x x}+u_{y y}+\frac{u_{x}^{2}+u_{y}^{2}}{1+u_{t}^{2}} u_{t t}+2 \frac{u_{y}-u_{x} u_{t}}{1+u_{t}^{2}} u_{x t}-2 \frac{u_{x}+u_{y} u_{t}}{1+u_{t}^{2}} u_{y t}=k \frac{\left(1+u_{t}^{2}+u_{x}^{2}+u_{y}^{2}\right)^{3 / 2}}{1+u_{t}^{2}},
$$

in the variables $(x, y, t) \in \mathbb{R}^{3}$. In $[6]$ it is pointed out that the principal part of the above operator can be written in the form $(2.3)$

$$
\mathcal{L} u=X^{2} u+Y^{2} u
$$


in terms of the nonlinear vector fields

$$
X=\partial_{x}+\frac{u_{y}-u_{x} u_{t}}{1+u_{t}^{2}} \partial_{t}, \quad Y=\partial_{y}-\frac{u_{x}+u_{y} u_{t}}{1+u_{t}^{2}} \partial_{t} .
$$

Then, based on the notion of "intrinsic" Taylor expansion of the coefficients of the operator, a modification of the freezing method used by Rothschild and Stein in [23] is developed.

Analogously, we remark that, by letting

$$
X_{j}=\partial_{x_{j}}, \quad j=1, \ldots, N-1 \text { and } X_{0}=u \partial_{y}-\partial_{t},
$$

then $L$ in (1.3) can be formally represented as

$$
L=\sum_{j=1}^{N-1} X_{j}^{2}+X_{0} .
$$

Since the commutator of $X_{j}$ and $X_{0}$ is

$$
\left[X_{j}, X_{0}\right]=\left(\partial_{x_{j}} u\right) \partial_{y}
$$

the Hörmander condition is satisfied if

$$
\partial_{x_{j}} u(x, y, t) \neq 0,
$$

for every $(x, y, t)$, for some index $j$. Note that the regularity of the solution $u$ in Theorem 2.1 is stated in condition (1.4), then $\partial_{x_{j}} u$ is defined almost everywhere and the above condition has to be considered only formally. Then we first state a regularity result for classical solutions to (1.3).

Theorem 2.2 Let $\Omega$ be an open set in $\mathbb{R}^{N+1}$ and $u$ a classical solution of (1.3) on $\Omega$ with $f \in C^{\infty}$. If (2.5) holds, then $u \in C^{\infty}(\Omega)$.

If we do not require any assumption on the commutators (in particular, if we do not require anymore condition (2.5)) then the Lie algebra associated to the operator is completely unknown. However we consider $L$ as a subelliptic operator with respect to some tentative Lie groups. This allows us to prove the existence of the derivatives $\partial_{x_{j} x_{j}} u$ and $X_{0} u$, defined as the directional derivative with respect to the vector $\nu_{z}=(0, u(z),-1)$ at the point $z=(x, y, t)$

$$
X_{0} u(z) \equiv \frac{\partial u}{\partial \nu_{z}}(z)=\lim _{h \rightarrow 0} \frac{u\left(z+h \nu_{z}\right)-u(z)}{h} .
$$

Then we have

Theorem 2.3 The strong solution u to (1.3) in Theorem 2.1 is a classical solution in the sense that $\partial_{x_{j} x_{j}} u, j=1, \ldots, N-1$, and $X_{0} u$ are continuous functions and the equation is satisfied at every point.

This result is quite reasonable, since without assuming the Hörmander condition we are able to prove the regularity of $u$ only in the directions of the vector fields. Note that, although the derivative $\frac{\partial u}{\partial \nu_{z}}$ can be obtained as a sum of more simple terms

$$
\frac{\partial u}{\partial \nu_{z}}=u \partial_{y} u-\partial_{t} u
$$

it is not true in general that the terms $u \partial_{y} u$ and $\partial_{t} u$ are continuous. Also note that, at this point, the Hörmander condition (2.5) is meaningful since $X_{j} u$ are defined and continuous functions. 


\section{Existence}

The proof of Theorem 2.1 is based on some estimates which can be obtained by adapting the classical Bernstein's method. We consider the regularized Cauchy-Dirichlet problem in a cylinder

$$
\begin{aligned}
L_{v}^{\varepsilon} u \equiv \triangle_{x} u+\varepsilon^{2} \partial_{y y} u+v \partial_{y} u-\partial_{t} u & =f(\cdot, v), & & \text { in } \left.Q_{T}=B \times\right] 0, T[, \\
u & =g, & & \text { in } \partial_{p} Q_{T} .
\end{aligned}
$$

where $\varepsilon>0, B$ is a ball in $\mathbb{R}^{N}$ and $\partial_{p} Q_{T}$ denotes the "parabolic" boundary of $Q_{T}$ defined as $(B \times\{0\}) \cup(\partial B \times[0, T])$. By a standard density argument we may assume that $f, g \in C^{\infty} \cap \operatorname{Lip}$. We fix a positive constant $c_{1}$ such that, for $(x, y, t, v) \in Q_{T}$, it holds

$$
\begin{aligned}
& c_{1} \geq \max \{\text { Lipschitz constants of } f, g\}, \\
& |g(x, y)| \leq c_{1} \sqrt{1+|(x, y)|^{2}}, \quad|f(x, y, t, v)| \leq c_{1} \sqrt{1+|(x, y, t, v)|^{2}} .
\end{aligned}
$$

Given $\alpha \in] 0,1\left[\right.$, we assume that the coefficient $v$ in (3.1)-(3.2) belongs to $C_{1+\alpha}\left(\bar{Q}_{T}\right)$, the Hölder space w.r.t. to the parabolic distance $d\left((x, y, t),\left(x^{\prime}, y^{\prime}, t^{\prime}\right)\right)=\left|x-x^{\prime}\right|+\left|y-y^{\prime}\right|+\left|t-t^{\prime}\right|^{\frac{1}{2}}$. Moreover we assume that $v$ satisfies in $Q_{T}$ the estimates

$$
\begin{aligned}
|v(x, y, t)| & \leq 2 c_{1} \sqrt{1+|(x, y)|^{2}}, \\
\left|\partial_{x_{i}} v\right| & \leq 2 c_{1}, \quad i=1, \ldots, N-1, \quad\left|\partial_{y} v\right| \leq 2 c_{1} .
\end{aligned}
$$

The following proposition is the key step in the proof of Theorem 2.1 which then follows by the Schauder's fixed point theorem and passing at limit as $\varepsilon$ goes to zero.

Proposition 3.1 There exists $T>0$ such that, under the above assumptions, every classical solution of (3.1)-(3.2) verifies the $\varepsilon$-uniform estimates (3.4), (3.5).

Proof Let $u$ be a classical solution of (3.1)-(3.2). We prove estimate (3.4) for $u$ by applying the maximum principle to the functions $H \pm u$ where $H$ is defined as follows

$$
H(x, y, t)=\left(c_{1}+\mu t\right) \sqrt{1+|(x, y)|^{2}}
$$

and $\mu$ is to be suitably fixed. Keeping in mind (3.3) and (3.4), is easily verified that

$$
L_{v}^{\varepsilon} H(x, y, t) \leq \frac{\left(1+\varepsilon^{2}\right)\left(c_{1}+\mu T\right)}{\sqrt{1+|(x, y)|^{2}}}+\left(\left(c_{1}+\mu T\right) c_{1}-\mu\right) \sqrt{1+|(x, y)|^{2}} \leq-|f(x, y, t, v(x, y, t))|,
$$

if $\mu, \frac{1}{T}$ are suitably large. On the other hand, by (3.3), $\left.H\right|_{\partial_{p} Q_{T}} \geq|g|$. Therefore, by the maximum principle, we infer that $H \pm u \geq 0$ on $Q_{T}$, that is

$$
|u| \leq H \leq 2 c_{1} \sqrt{1+|(x, y)|^{2}}, \quad \text { if } T \leq \frac{c_{1}}{\mu} .
$$


Next we prove the estimate for the $y$-derivative of $u$. We differentiate equation (3.1) w.r.t. the variable $y$ and then multiply it by $e^{-2 \lambda t} \partial_{y} u$. Denoting $\omega=\left(e^{-\lambda t} \partial_{y} u\right)^{2}$, we obtain

$$
\begin{aligned}
L_{v}^{\varepsilon} \omega & =e^{-2 \lambda t} L_{v}^{\varepsilon}\left(\partial_{y} u\right)^{2}+2 \lambda \omega \\
& =2\left(e^{-2 \lambda t}\left(\left|\nabla_{x} \partial_{y} u\right|^{2}+\varepsilon^{2}\left(\partial_{y y} u\right)^{2}+\partial_{y} u\left(\partial_{y} f+\partial_{v} f \partial_{y} v\right)\right)+\lambda \omega\right) \\
& \geq 2\left(e^{-2 \lambda t} \partial_{y} u\left(\partial_{y} f+\partial_{v} f \partial_{y} v\right)+\lambda \omega\right) .
\end{aligned}
$$

Hence, by setting $w=\omega-c_{1}^{2}$, we get from (3.6)

$$
L_{v}^{\varepsilon} w \geq 2 \sqrt{\omega}\left(-\left|\partial_{y} f\right|-\left|\partial_{y} v \partial_{v} f\right|+\lambda \sqrt{\omega}\right)
$$

(by $(3.3),(3.5)$ and by the elementary inequality $\sqrt{\omega} \geq \frac{\sqrt{2}}{2}\left(c_{1}+\operatorname{sgn}(w) \sqrt{|w|}\right)$ )

$$
\geq \sqrt{2 \omega}\left(c_{1}\left(\lambda-2 \sqrt{2} c_{1}-\sqrt{2}\right)+\lambda \operatorname{sgn}(w) \sqrt{|w|}\right)
$$

(for $\lambda=\lambda\left(c_{1}\right)$ suitable large)

$$
\geq c \sqrt{\omega|w|} \operatorname{sgn}(w)
$$

for some positive constant $c=c\left(c_{1}\right)$. By contradiction, we want to prove that $w \leq 0$ in $Q_{T}$. It will follow that

$$
\left|\partial_{y} u\right| \leq c_{1} e^{\lambda t}
$$

which implies (3.6) if $T=T\left(c_{1}\right)>0$ is sufficiently small. Let $z_{0}$ be the maximum of $w$ on $\bar{Q}_{T}$. If $w\left(z_{0}\right)>0$, then $z_{0} \in Q_{T} \backslash \partial_{p} Q_{T}$, since by construction $w \leq 0$ on $\partial_{p} Q_{T}$. This leads to a contradiction since, by $(3.7)$

$$
0 \geq L_{v}^{\varepsilon} w\left(z_{0}\right) \geq c \sqrt{\omega\left(z_{0}\right) w\left(z_{0}\right)}>0
$$

By a similar technique, we prove estimate (3.5) of the $x$-derivatives of $u$. We set

$$
\omega=\left(e^{-\lambda t} \partial_{x_{k}} u\right)^{2}, \quad w=\omega-c_{1}^{2} .
$$

Differentiating equation (3.1) w.r.t. $x_{k}$ and multiplying it by $e^{-2 \lambda t} \partial_{x_{k}} u$, we get

$$
L_{v}^{\varepsilon} w=e^{-2 \lambda t} L_{v}^{\varepsilon}\left(\partial_{x_{k}} u\right)^{2}+2 \lambda \omega=2\left(e^{-2 \lambda t} \partial_{x_{k}} u\left(\partial_{x_{k}} f+\partial_{x_{k}} v \partial_{v} f\right)+\lambda \omega\right)
$$

(by $(3.3),(3.5)$, and the estimate of $\partial_{y} u$ just proved)

$$
\geq \sqrt{2 \omega}\left(c_{1}\left(\lambda-\sqrt{2}-2 \sqrt{2} c_{1}-4 \sqrt{2} c_{1}^{2}\right)+\lambda \operatorname{sgn}(w) \sqrt{|w|}\right)
$$

(if $\lambda=\lambda\left(c_{1}\right)$ is suitable large)

$$
\geq c \sqrt{\omega|w|} \operatorname{sgn}(w)
$$

for some positive constant $c$ which depends only on $c_{1}$. As before, we infer that $w \leq 0$ which yields (3.5). 


\section{Regularity}

Theorems 2.2 and 2.3 rely on some representation formulas for $u$ and its derivatives in terms of the fundamental solution of a frozen operator.

The freezing method is a well-known technique, classically used to study the regularity of solutions to linear parabolic equations. In this case, the associated frozen operator is simply obtained by evaluating the coefficients at a fixed point. This new operator is, up to a linear change of coordinates, the heat operator and its fundamental solution can be considered as a parametrix of the fundamental solution of the non-constant coefficients operator. A much more difficult argument was used to prove the existence of a fundamental solution for Hörmander type operators (2.3). Indeed the frozen vector fields

$$
X_{i, x_{0}}=\sum_{i=1}^{n} a_{i j}\left(x_{0}\right) \partial_{x_{j}} \quad i=0, \cdots, p
$$

commute, and the generated Lie algebra has dimension, in general, less than $n$. In this case the operator

$$
\sum_{i=1}^{p} X_{i, x_{0}}^{2}-X_{0, x_{0}}
$$

is not hypoelliptic, and it has not a fundamental solution. Folland and Stein [14] first pointed out that the model operators in this case are operators of the form (2.3) such that the Lie algebra generated by $X_{0}, \ldots, X_{p}$ is nilpotent and stratified. Later on Rothschild and Stein introduced in [23] an abstract and very general version of the freezing method. The choice of the frozen vector fields $X_{i, x_{0}}$ is made in such a way that their generated Lie algebra has, at low orders, the same structure as $\operatorname{Lie}\left(X_{0}, \ldots, X_{p}\right)$. With this choice of vector fields, the operator in (4.1) is hypoelliptic and its fundamental solution $\Gamma_{x_{0}}$ is a parametrix for (2.3). As said above, we employ here a modification of this technique, introduced by Citti in [6].

We define a frozen operator in terms of the notion of "intrinsic" Taylor expansion of the coefficients. We first consider $L$ as a linearized operator

$$
L_{u}=\sum_{j=1}^{N-1} X_{j}^{2}+X_{0},
$$

where $X_{j}=\partial_{x_{j}}$ and $X_{0}=u \partial_{y}-\partial_{t}$. Then we let, for every $z_{0}=\left(x_{0}, y_{0}, t_{0}\right) \in \mathbb{R}^{N+1}$,

$$
L_{z_{0}}=\sum_{j=1}^{N-1} X_{j}^{2}+X_{0, z_{0}},
$$

where $X_{0, z_{0}} \equiv\left(u\left(z_{0}\right)+\left(x-x_{0}\right) \cdot \nabla_{x} u\left(z_{0}\right)\right) \partial_{y}-\partial_{t}$. Under the assumptions of Theorem 2.2, this choice ensures that the frozen operator $L_{z_{0}}$ is a nilpotent Hörmander type operator, it has a fundamental solution $\Gamma_{z_{0}}$ and an associated control distance $d_{z_{0}}$. For simplicity we assume that $f$ does not depend on $u$. We represent the solution $u$ in terms of $\Gamma_{z_{0}}$ :

$$
u(z)=\int \Gamma_{z_{0}}(z, \zeta) L_{z_{0}} u(\zeta) d \zeta=\int \Gamma_{z_{0}}(z, \zeta) f(\zeta) d \zeta+\int \Gamma_{z_{0}}(z, \zeta) K_{z_{0}}(z, \zeta) d \zeta
$$


where

$$
K_{z_{0}}(z, \zeta) \equiv\left(L_{u}-L_{z_{0}}\right) u(\zeta)=\left(u(\zeta)-u\left(z_{0}\right)-\left(\xi-x_{0}\right) \cdot \nabla_{x} u\left(z_{0}\right)\right) \partial_{y} u(\zeta) .
$$

Since $L_{u}$ is a second order operator, we have to consider the term $X_{0, z_{0}} u$ as a second order derivative, whereas $X_{j}$ is a first order derivative. As a consequence, the first order Taylor polynomial of $u$, with initial point at $z_{0}$, is given by

$$
P_{z_{0}}^{1} u(z)=u\left(z_{0}\right)+\left(x-x_{0}\right) \cdot \nabla_{x} u\left(z_{0}\right) .
$$

Now, by (1.4), $\partial_{y} u$ is bounded so, when $u \in C^{1, \alpha}$, we have

$$
K_{z_{0}}(z, \zeta)=O\left(d\left(z_{0}, \zeta\right)^{1+\alpha}\right) \quad \text { as } \quad d\left(z_{0}, \zeta\right) \rightarrow 0 .
$$

By choosing $z_{0}=z$, this estimate allows us to differentiate up to 3 times the above representation formula under the integral sign:

$$
D^{3} u\left(z_{0}\right)=\int D \Gamma_{z_{0}}\left(z_{0}, \zeta\right) D^{2} f(\zeta) d \zeta+\int D^{3} \Gamma_{z_{0}}\left(z_{0}, \zeta\right) K_{z_{0}}\left(z_{0}, \zeta\right) d \zeta
$$

and conclude that $u \in C^{3, \alpha}$. A rather delicate argument, based on the use of some high order difference quotients allows us to iterate this argument and conclude the proof of Theorem 2.2.

The proof of Theorem 2.3 follows the same lines, but there is another difficulty. Indeed, without the assumption of the Hörmander condition (2.5), the frozen operator $L_{z_{0}}$ may not have a fundamental solution. In this case, it is convenient to approximate $X_{0}$ by the vector field

$$
\widetilde{X}_{0, z_{0}}=\left(u\left(z_{0}\right)+\left(x-x_{0}\right)_{1}\right) \partial_{y}-\partial_{t},
$$

where $x_{1}$ denotes the first component of the vector $x$. Then the operator

$$
\widetilde{L}_{z_{0}}=\sum_{j=1}^{N-1} X_{j}^{2}+\widetilde{X}_{0, z_{0}}
$$

does not depend on $\nabla_{x} u\left(z_{0}\right)$ and it is hypoelliptic. Note that, with respect to $X_{0, z_{0}}$, this vector field gives a less close approximation for $X_{0}$, since

$$
X_{0} u(z)-\tilde{X}_{0, z_{0}} u(z)=\left(u(z)-u\left(z_{0}\right)-\left(x-x_{0}\right)_{1}\right) \partial_{y} u(z)=O\left(d\left(z_{0}, z\right)\right),
$$

as $d\left(z_{0}, z\right) \rightarrow 0$, however it is sufficiently accurate to prove Theorem 2.2.

\section{References}

[1] Antonelli, F.; Barucci, E.; Mancino, M.E., A Comparison result for FBSDE with Applications to Decisions Theory. Math. Methods Oper. Res., in press (downloadble from http://www.dm.unibo.it/ pascucci/web/Ricerca/Ricerca.html).

[2] Antonelli, F.; Pascucci, A. On the viscosity solutions of a stochastic differential utility problem. J. Differential Equations, in press (downloadble from http://www.dm.unibo.it/ pascucci/web/Ricerca/Ricerca.html). 
[3] BARles, G., A weak Bernstein method for fully non-linear elliptic equations, Diff. Int. Eq., 4-2, (1991), 241-262

[4] Bian, B.; Dong, G., The regularity of viscosity solutions for a class of fully nonlinear equations, Sci. China, Ser. A 34, No.12, 1448-1457 (1991).

[5] Cabre, X.; Caffarelli, L. A., Fully nonlinear elliptic equations, Colloquium Publications. American Mathematical Society. 43. Providence, RI (1995).

[6] Citti, G., $C^{\infty}$ regularity of solutions of a quasilinear equation related to the Levi operator, Ann. Sc. Norm. Super. Pisa, Cl. Sci., IV. Ser. 23, No.3, 483-529 (1996).

[7] Citti, G.; Pascucci, A.; Polidoro, S., On the regularity of solutions to a nonlinear ultraparabolic equation arising in mathematical finance, Diff. Int. Eq. 14-6 701-738 (2001).

[8] Citti, G.; Pascucci, A.; Polidoro, S. Regularity properties of viscosity solutions of a non-Hörmander degenerate equation, J. Math. Pures Appl. 2001, 80 (9), 901-918 (2001).

[9] Crandall, M. G.; Ishit, H.; Lions, P.-L.,User's guide to viscosity solutions of second order partial differential equations, Bull. Am. Math. Soc., New Ser. 27, No.1, 1-67 (1992).

[10] L. Epstein, S. ZIN, Substitution, risk aversion and the temporal behavior of consumption and asset returns: a theoretical framework, Econometrica, 57 (1989), 937-969.

[11] Escobedo, M.; Vazquez, J.L.; ZuazuA, E., Entropy solutions for diffusion-convection equations with partial diffusivity, Trans. Am. Math. Soc. 343, No.2, 829-842 (1994).

[12] W. H. Fleming, H. M. Soner, Controlled Markov processes and viscosity solutions, Applications of Mathematics, 25, 1993, Springer-Verlag.

[13] Folland, G.B., Subelliptic estimates and function spaces on nilpotent Lie groups, Ark. Mat. 13, 161-207 (1975).

[14] Folland, G.B.; Stein, E.M., Estimates for the $\bar{\partial}_{b}$ complex and analysis on the Heisenberg group, Commun. Pure Appl. Math. 27, 429-522 (1974).

[15] B. FRANChi, E. LANCONELli, Hölder regularity theorem for a class of linear non-uniformly elliptic operators with measurable coefficients, Ann. Sc. Norm. Super. Pisa, Cl. Sci., IV. Ser. 10 (1983), 523-541.

[16] HöRmander, L., Hypoelliptic second order differential equations, Acta Math. 119, 147-171 (1967).

[17] Jerison, D.S.; SAnchez-CAlle, A., Estimates for the heat kernel for a sum of squares of vector fields, Indiana Univ. Math. J. 35, 835-854 (1986).

[18] Krylov, N.V., Hölder continuity and $L_{p}$ estimates for elliptic equations under general Hörmander's condition, Topol. Methods Nonlinear Anal. 9, No.2, 249-258 (1997).

[19] Moser, J., On Harnack's theorem for elliptic differential equations, Comm. Pure Appl. Math. 14, (1961).

[20] Nagel, A.; Stein, E.M.; Wainger, S., Balls and metrics defined by vector fields I: basic properties, Acta Math. 155, 103-147 (1985).

[21] PAscuCcI, A., Hölder regularity for a Kolmogorov equation, preprint

[22] Pascucci, A.; Polidoro S., On the Cauchy problem for a nonlinear ultraparabolic equation, preprint

[23] Rothschild, L.P.; Stein, E.M., Hypoelliptic differential operators on nilpotent groups, Acta Math. 137(1976), 247-320 (1977). 
[24] Trudinger, N.S., Hölder gradient estimates for fully nonlinear elliptic equations, Proc. R. Soc. Edinb., Sect. A 108, No.1/2, 57-65 (1988).

[25] Wang, L., On the regularity theory of fully nonlinear parabolic equations I and II, Commun. Pure Appl. Math. 45, No.1, 27-76 and No.2, 141-178 (1992). 